\title{
IIC
}

\section{Solving the Urban Positioning Problem using 3D-Mapping-Aided GNSS}

\section{Dr Paul D Groves}

Space Geodesy \& Navigation Laboratory University College London (p.groves@ucl.ac.uk)

ION GNSS+ 2016

14 September 2016

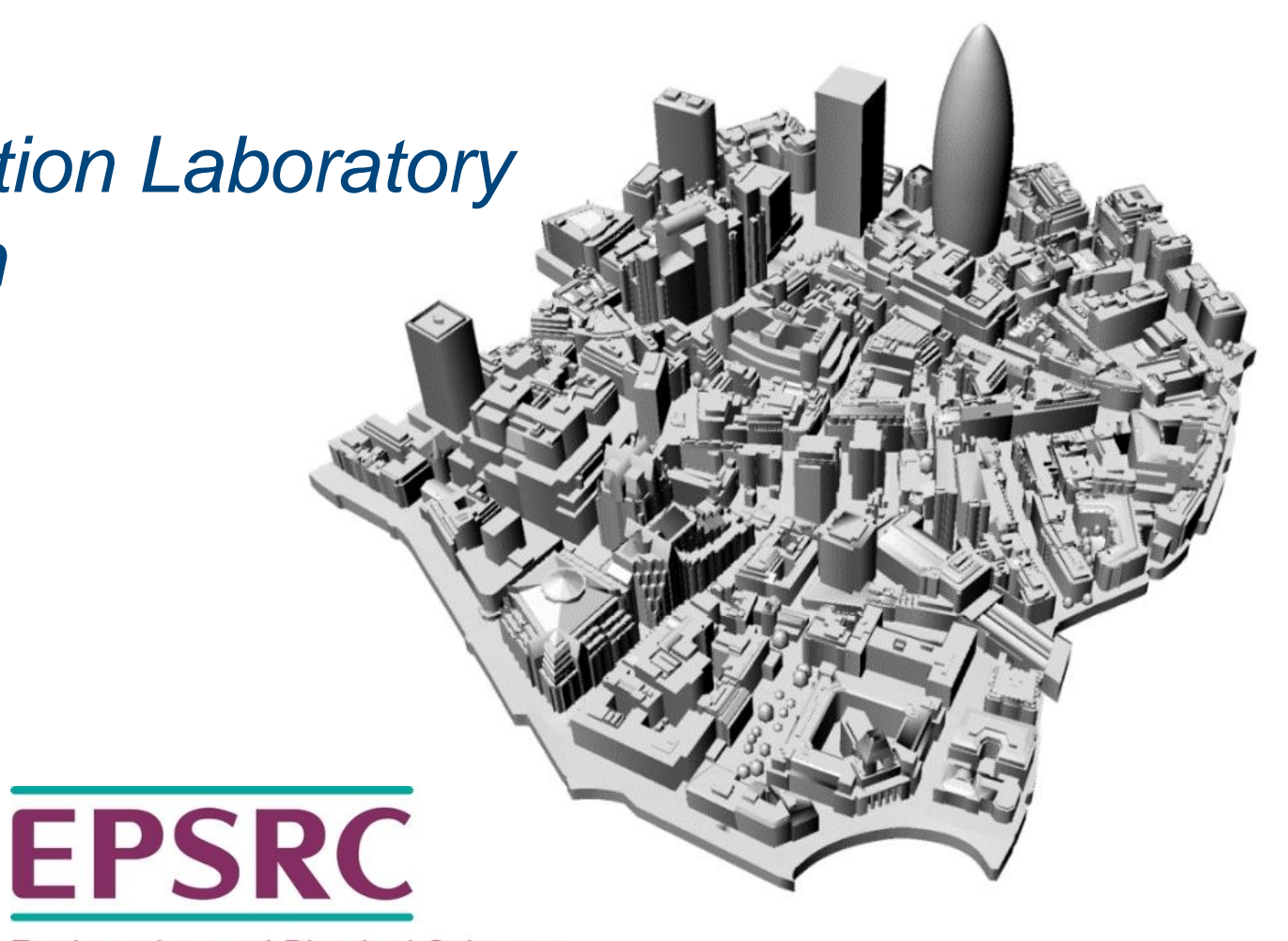




\section{Urban GNSS: Without 3D Mapping}

Reflected signal (path delay causes positioning error)

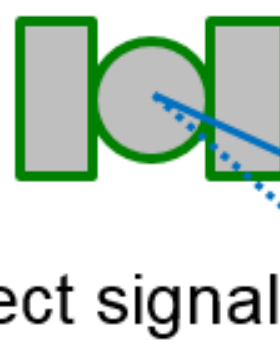
is blocked, but we don't know this

Position errors of 10-50 $\mathrm{m}$ are common

Direct signal

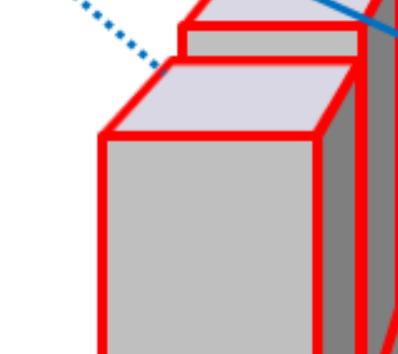

Directly-received signal (good for positioning), but we don't know this. we don't know this.
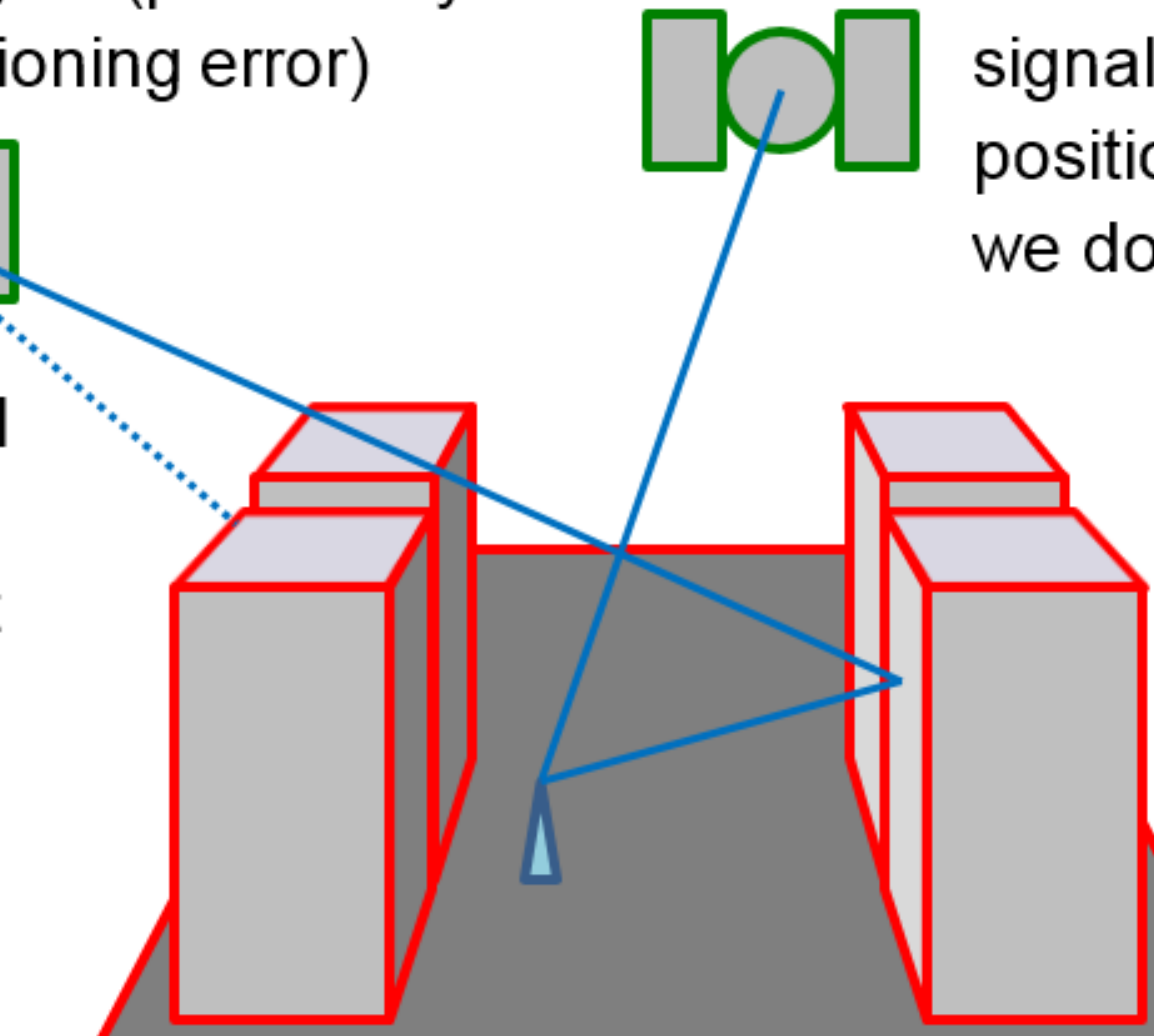

Unknown buildings

Terrain height unknown

28 m RMS horizontal (2D) Single-epoch 


\section{Urban GNSS: With 3D Mapping}

Signal known to be reflected:

Process accordingly

Position errors

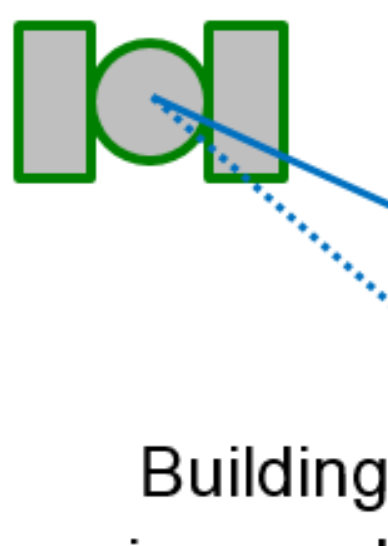

of a few meters

are common

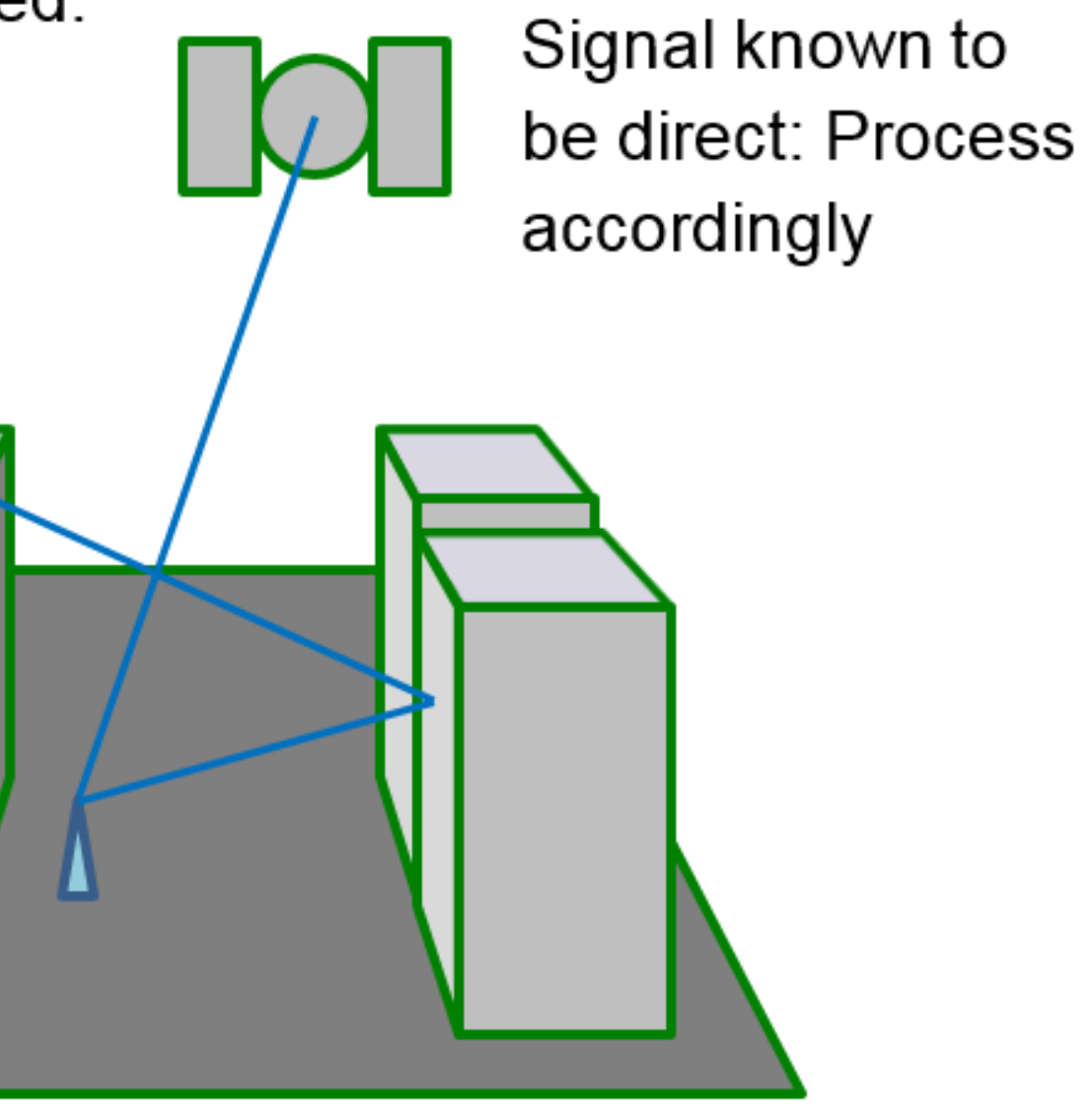

Known terrain height: Can aid positioning

4 m RMS horizontal (2D) Single-epoch 


\section{The Opportunity}

We Need

\section{Measurements}

\section{D Mapping}

\section{Algorithms}

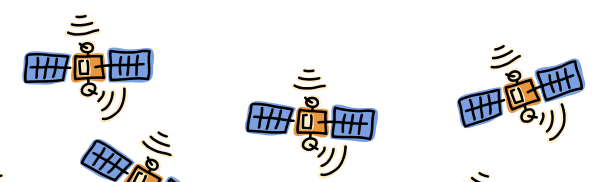

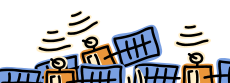

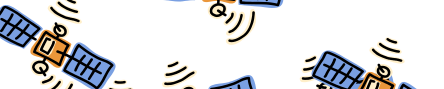

Q, 开)

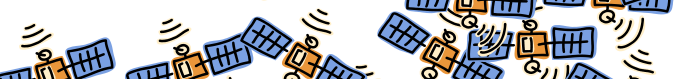

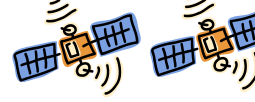
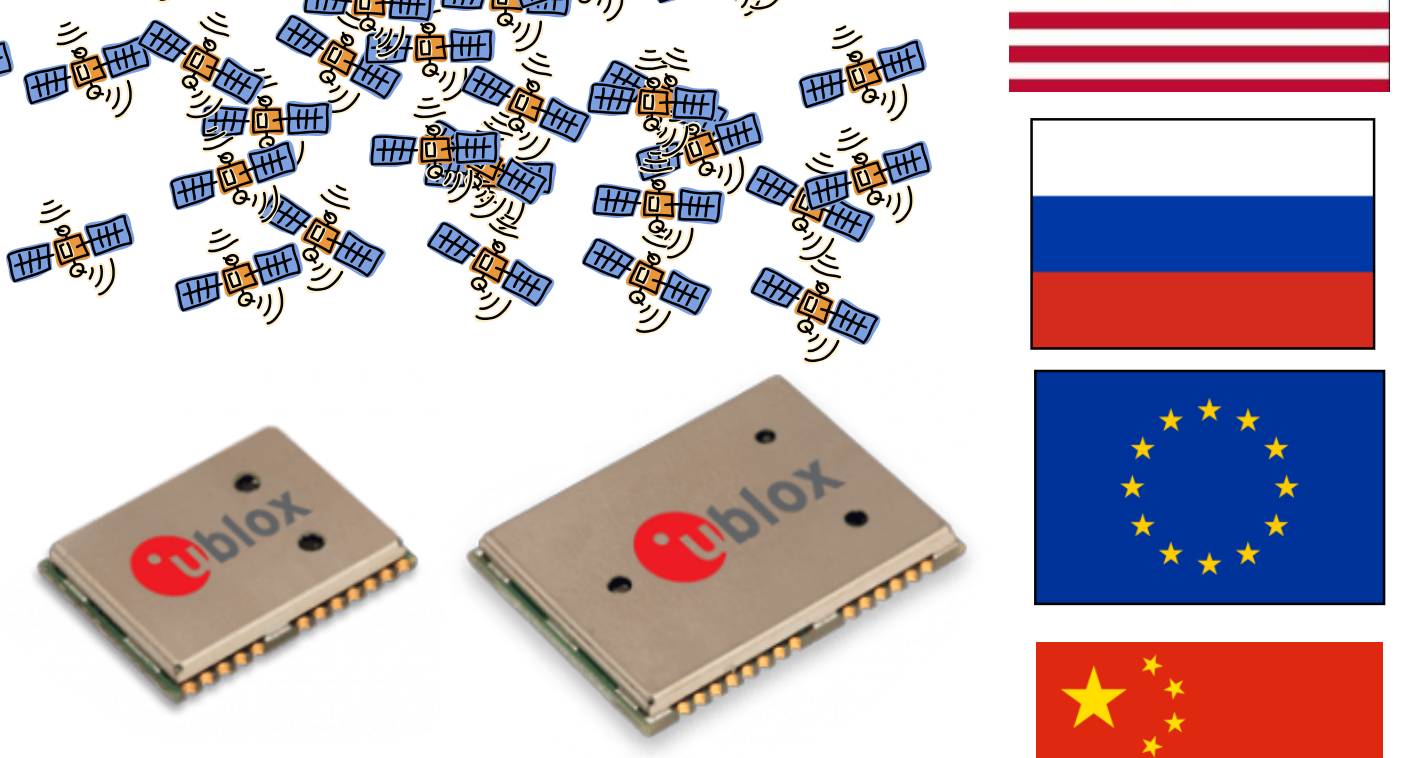

$\lambda^{*} *^{*}$ 


\section{The Opportunity}

\section{We Need}

Measurements

\section{D Mapping}

Algorithms

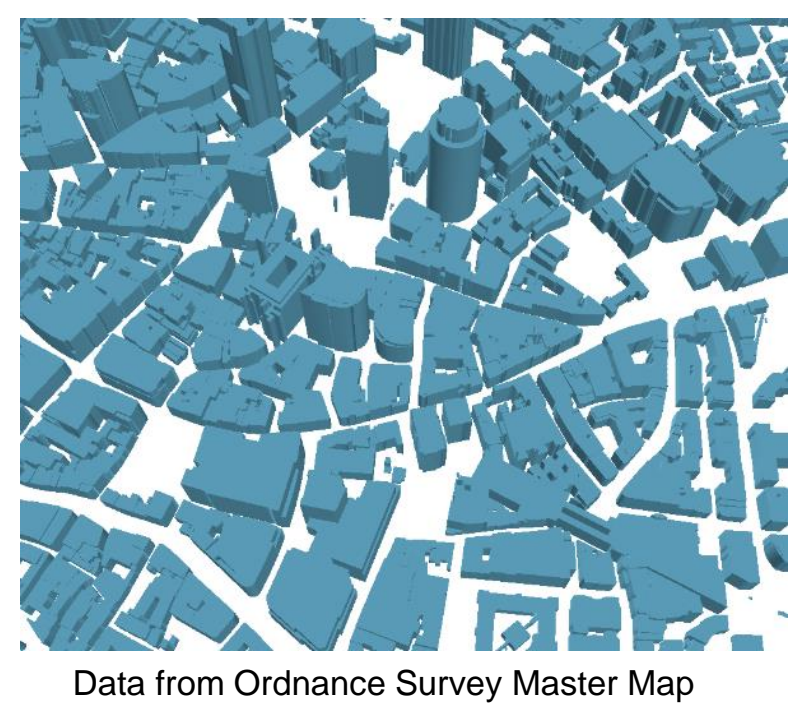

Simple 'block' maps are sufficient Open Street Map

National Mapping Agencies

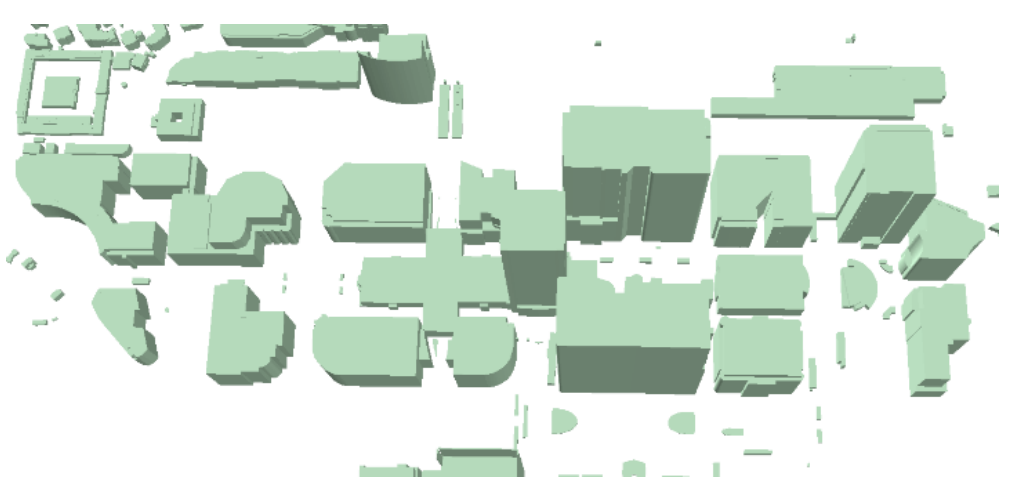

Data from Ordnance Survey Master Map 


\section{$\pm / C L_{1}$}

\section{The Opportunity}

We Need

\section{Measurements}

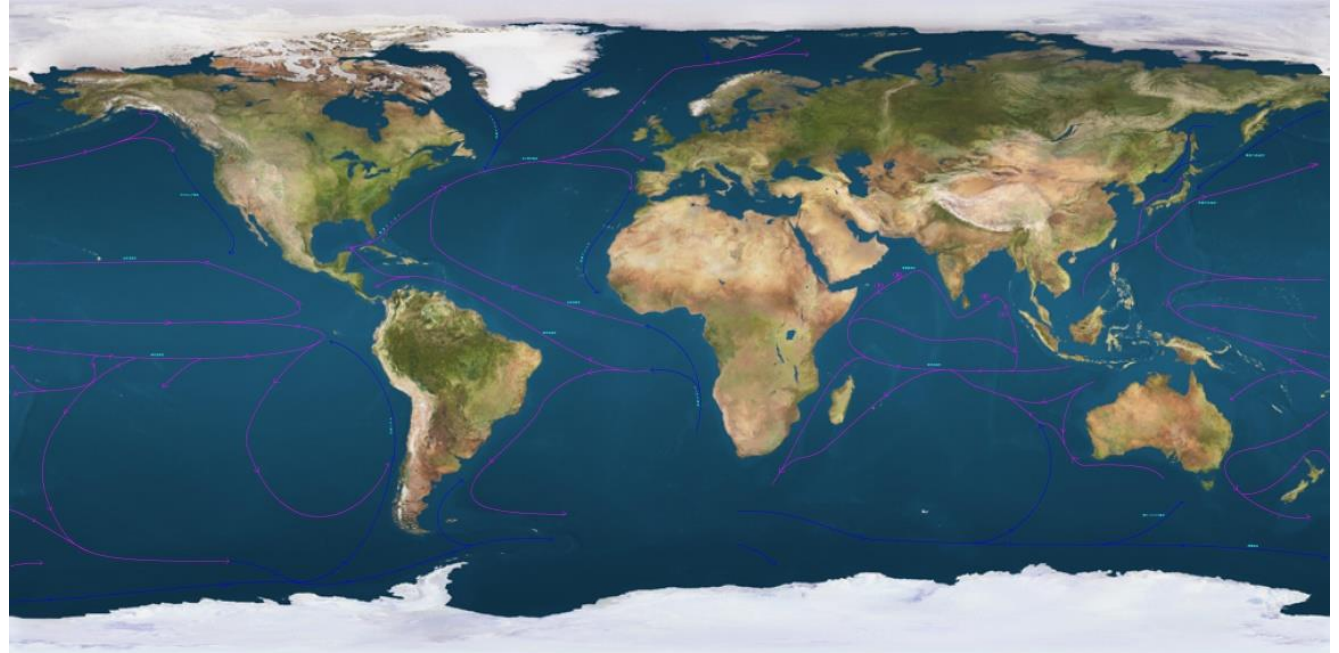

\section{D Mapping}

Algorithms
Lots of research has been done since 2010

$\sim 10$ Research groups in Europe, Asia, North America \& Middle East More than 50 papers published 


\section{D Mapping Aids GNSS in 3 Ways}

Height Aiding

Mappingaided Ranging

Shadow Matching
Signal known to be reflected:

Process accordingly

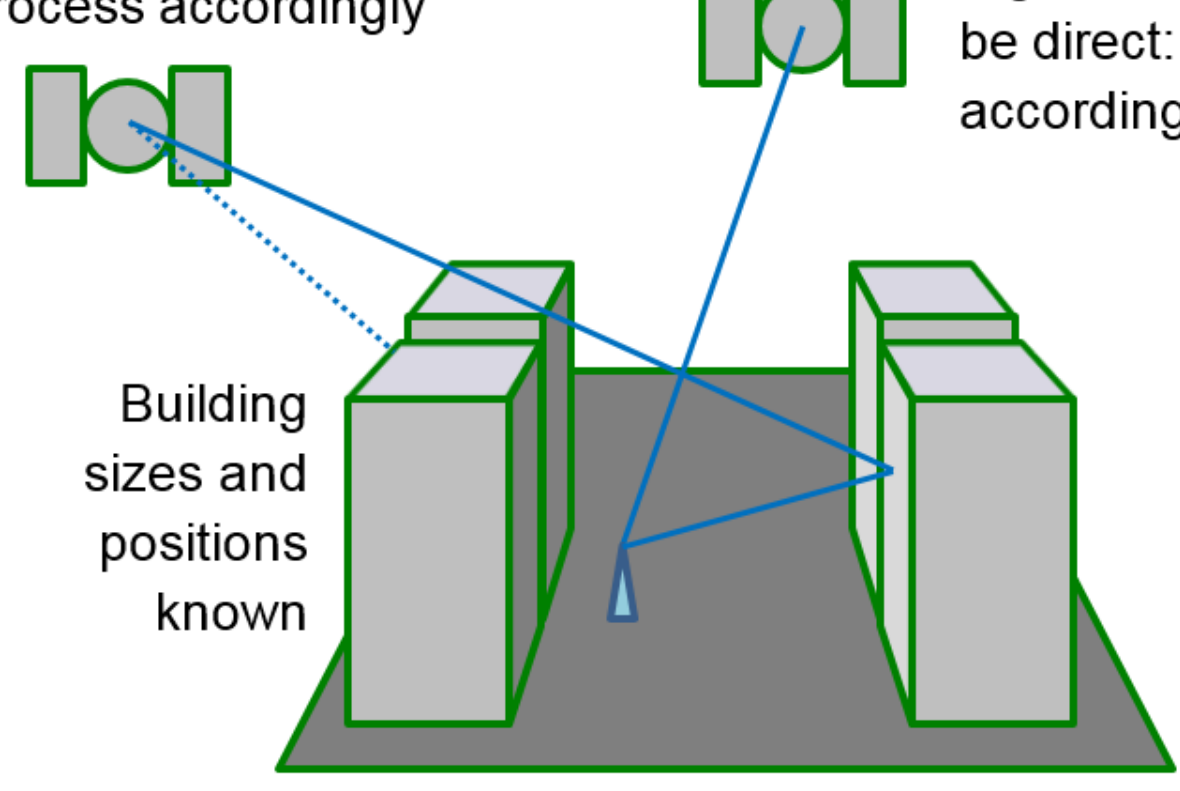

Known terrain height: Can aid positioning
Signal known to be direct: Process accordingly 


\section{Terrain Height Aiding}

In an open environment, this only improves vertical positioning

Where signal geometry is poor, horizontal positioning is nearly twice as accurate with height aiding

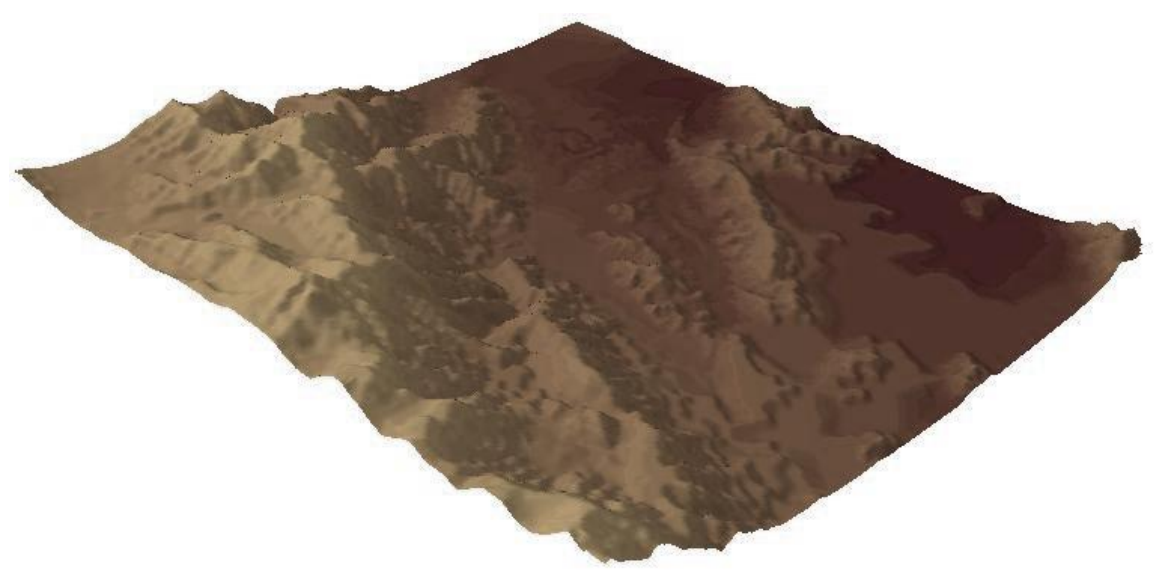

GNSS SVs

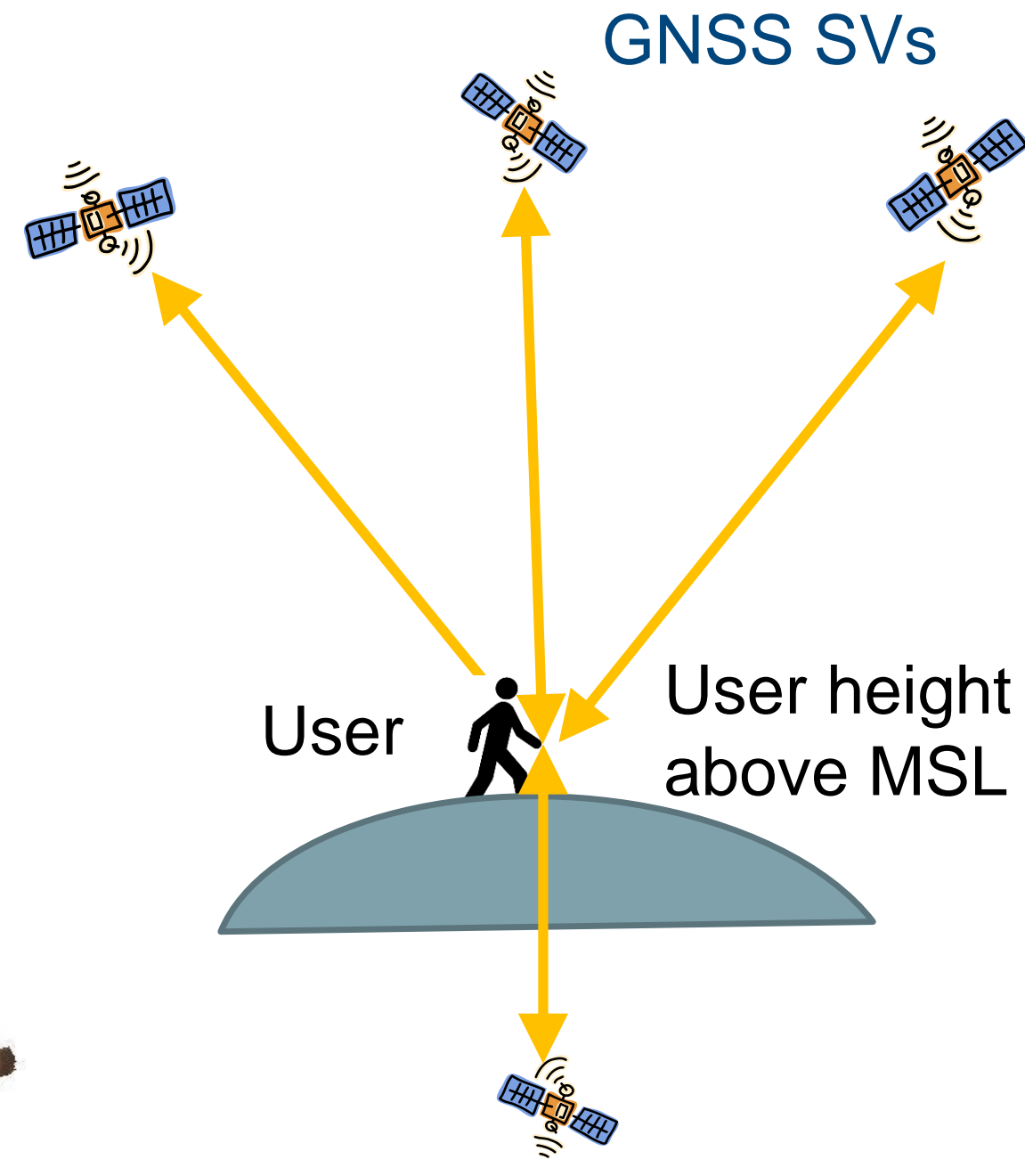

Virtual SV at centre of Earth 


\section{Non-line-of-sight (NLOS) Prediction}

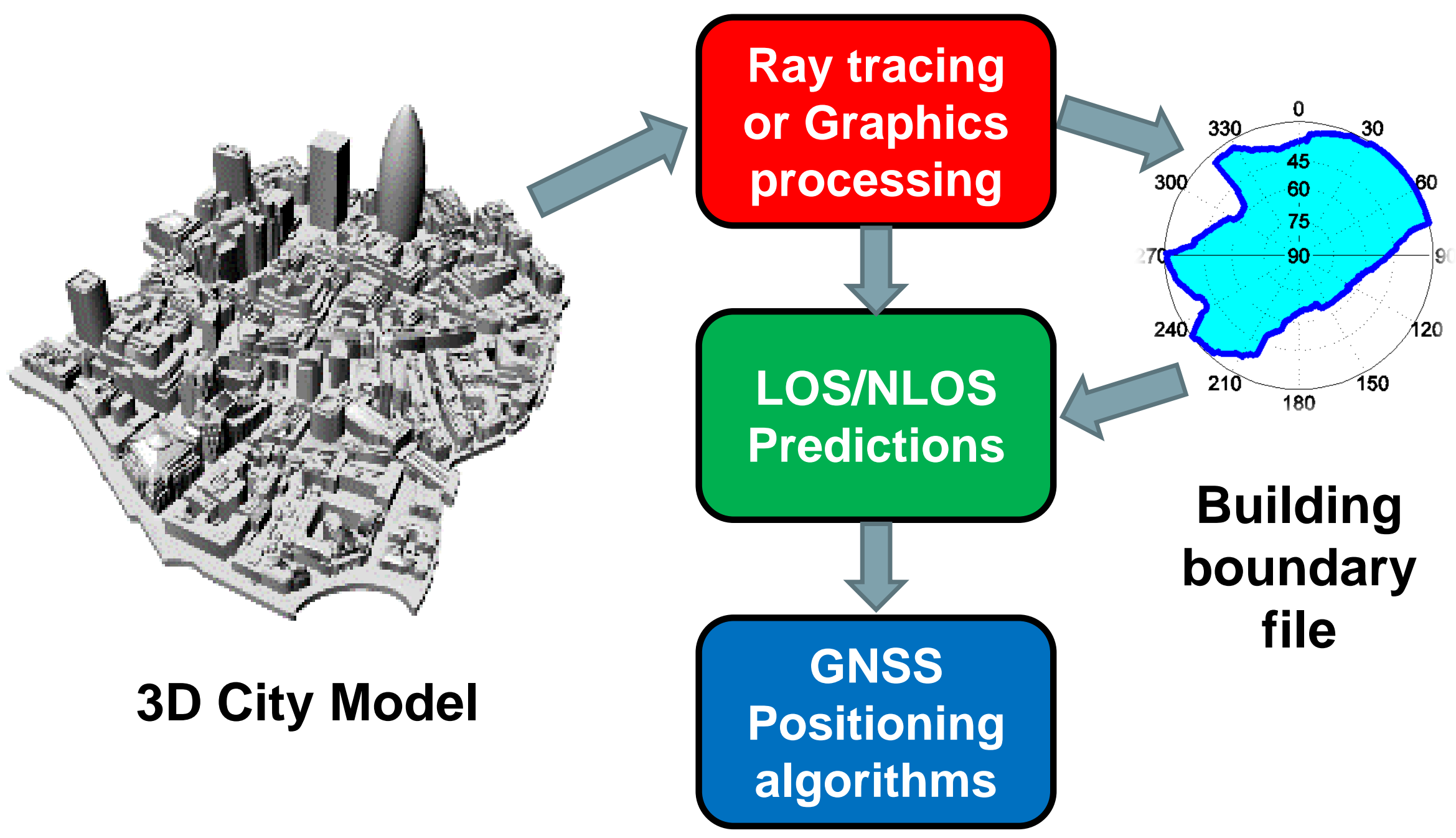




\section{D-Mapping-Aided GNSS Ranging}

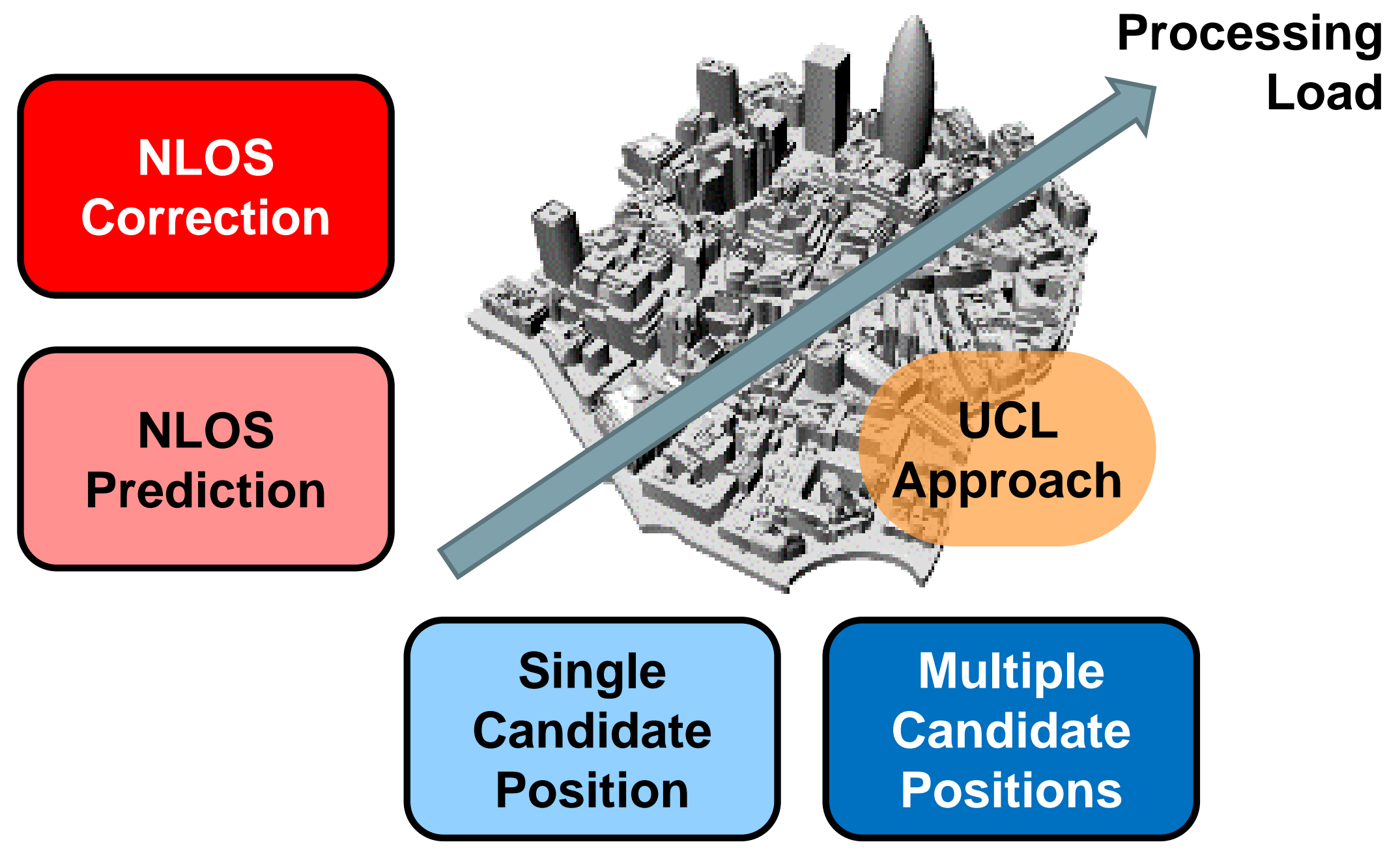

Other groups have implemented other approaches 


\section{Shadow Matching}

Conventional across-street positioning is poor

[Q⿻]

$\therefore$ We need a new approach...

No direct signal Direct signal received: user received: is here

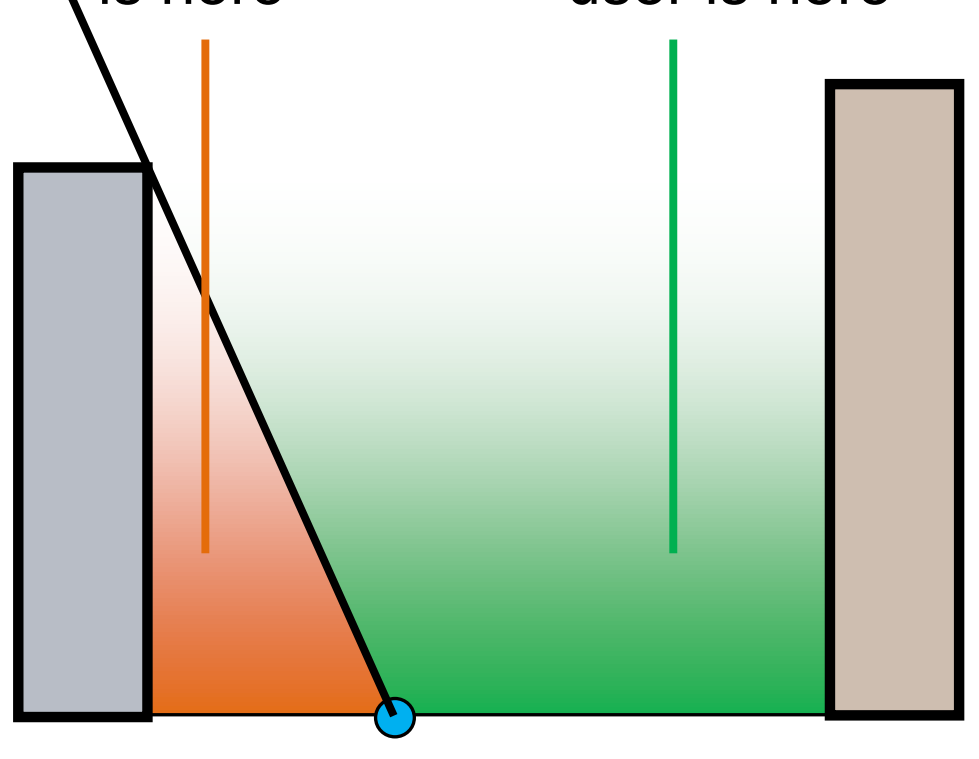

Direct signal received: user is here is here

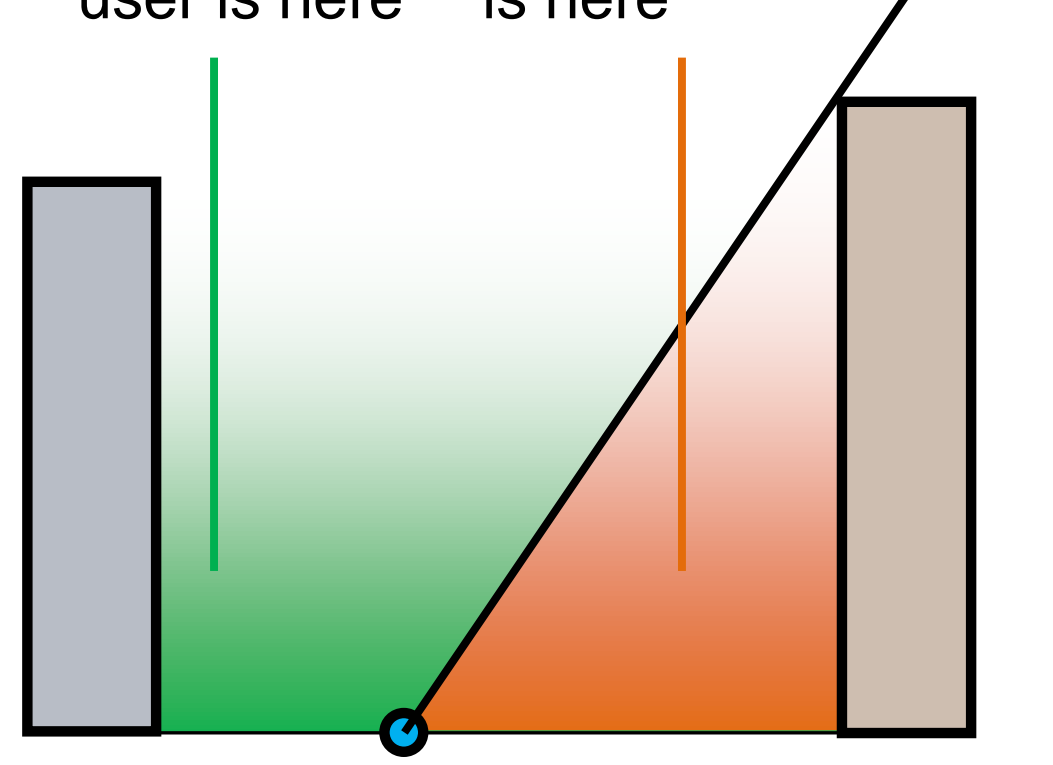


Single Epoch versus Multiple Epochs

\section{Single-epoch GNSS}

Few alternatives to $3 D$ mapping aiding

Multi-epoch GNSS

Many alternatives :

- Carrier smoothing

- Filtering (EKF)

- Integration with inertial and other DR sensors

- RTK carrier-phase positioning (potentially)

- Advanced signal processing techniques

But, these techniques need accurate initialization 3D-mapping-aided GNSS provides this in urban areas

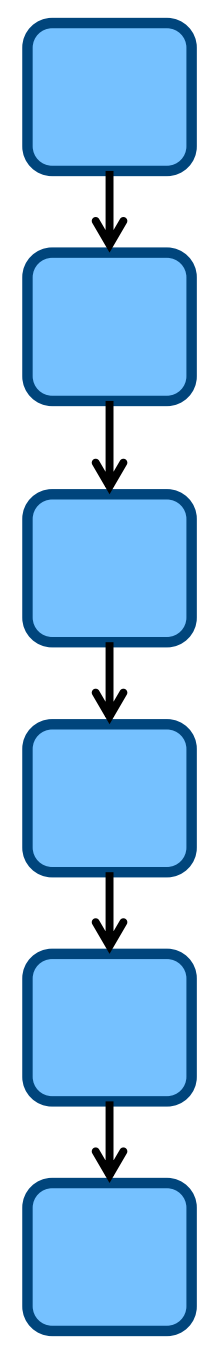




\section{Intelligent Urban Positioning: Concept}

Best performance is obtained by combining everything

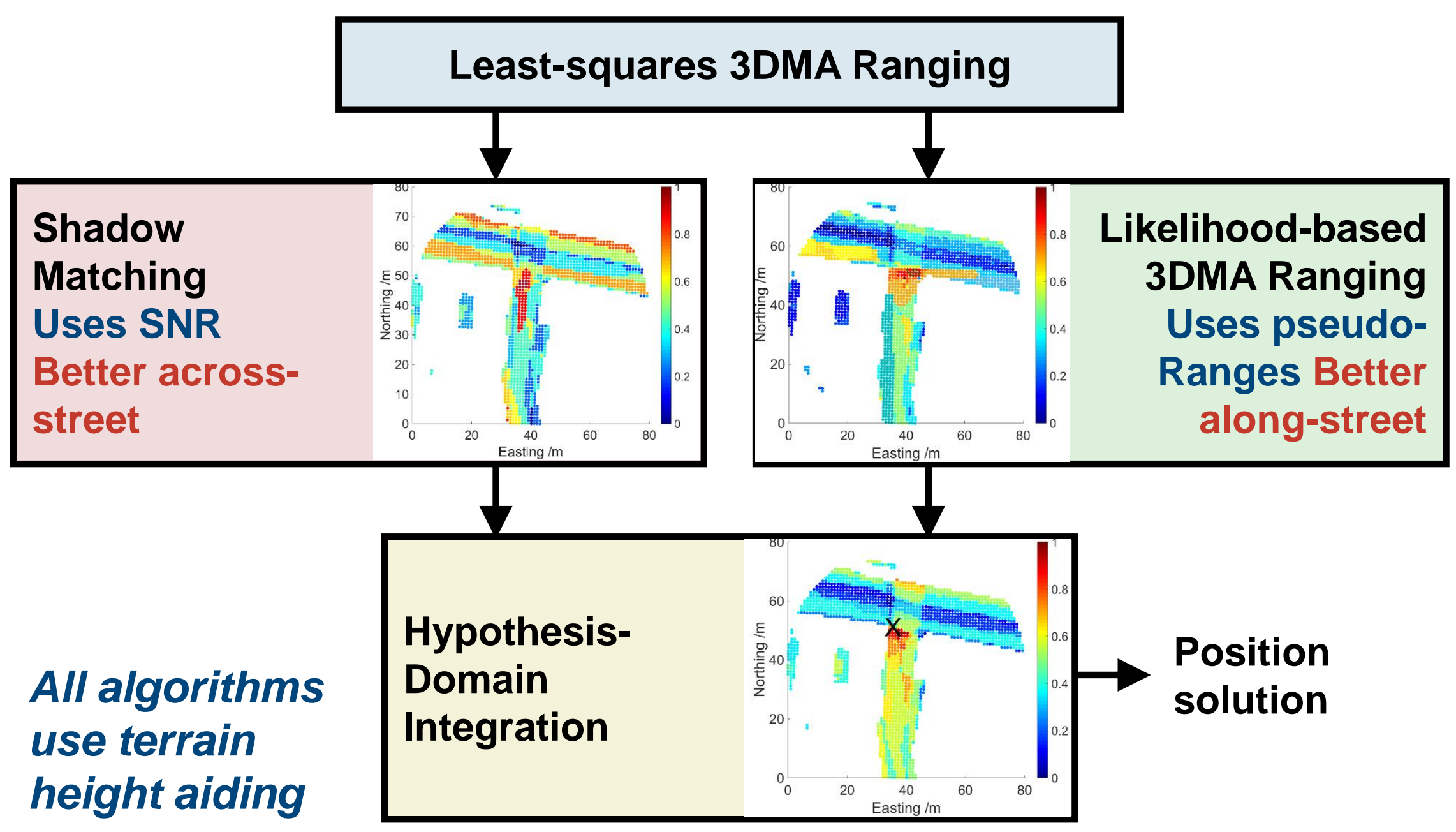




\section{IJCI}

\section{Intelligent Urban Positioning: Results (1)}
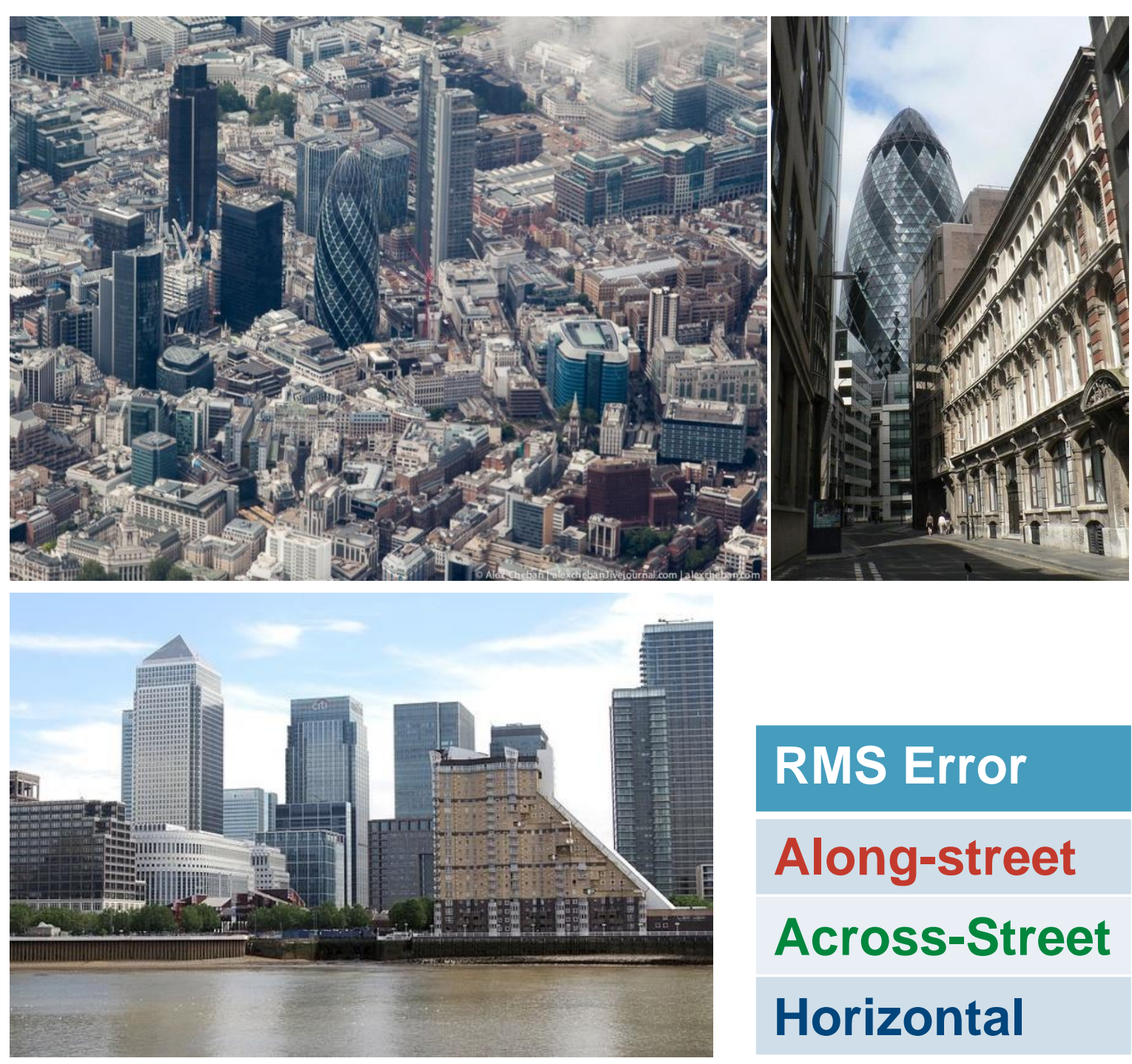

U-blox EVK M8T Single-epoch positioning

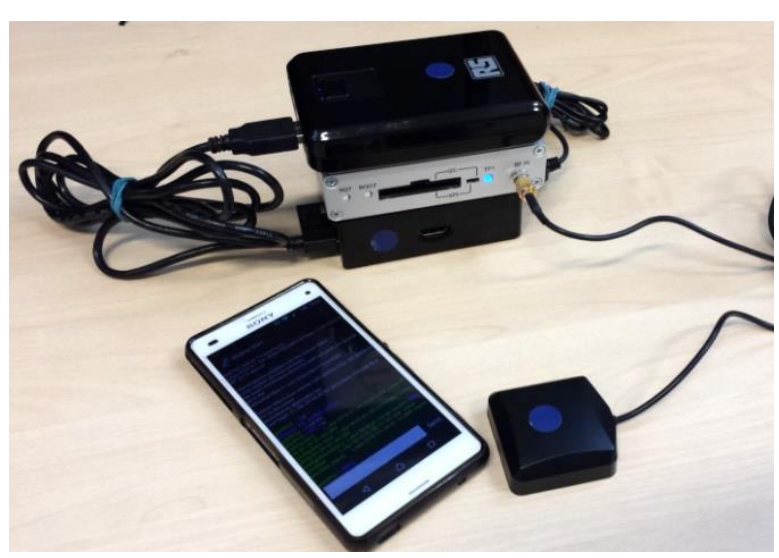

RMS Error Conventional IUP

Along-street

Across-Street

Horizontal
$13.5 \mathrm{~m}$

$24.7 \mathrm{~m}$

$28.2 \mathrm{~m}$
$2.9 \mathrm{~m}$

$2.8 \mathrm{~m}$

$4.0 \mathrm{~m}$ 

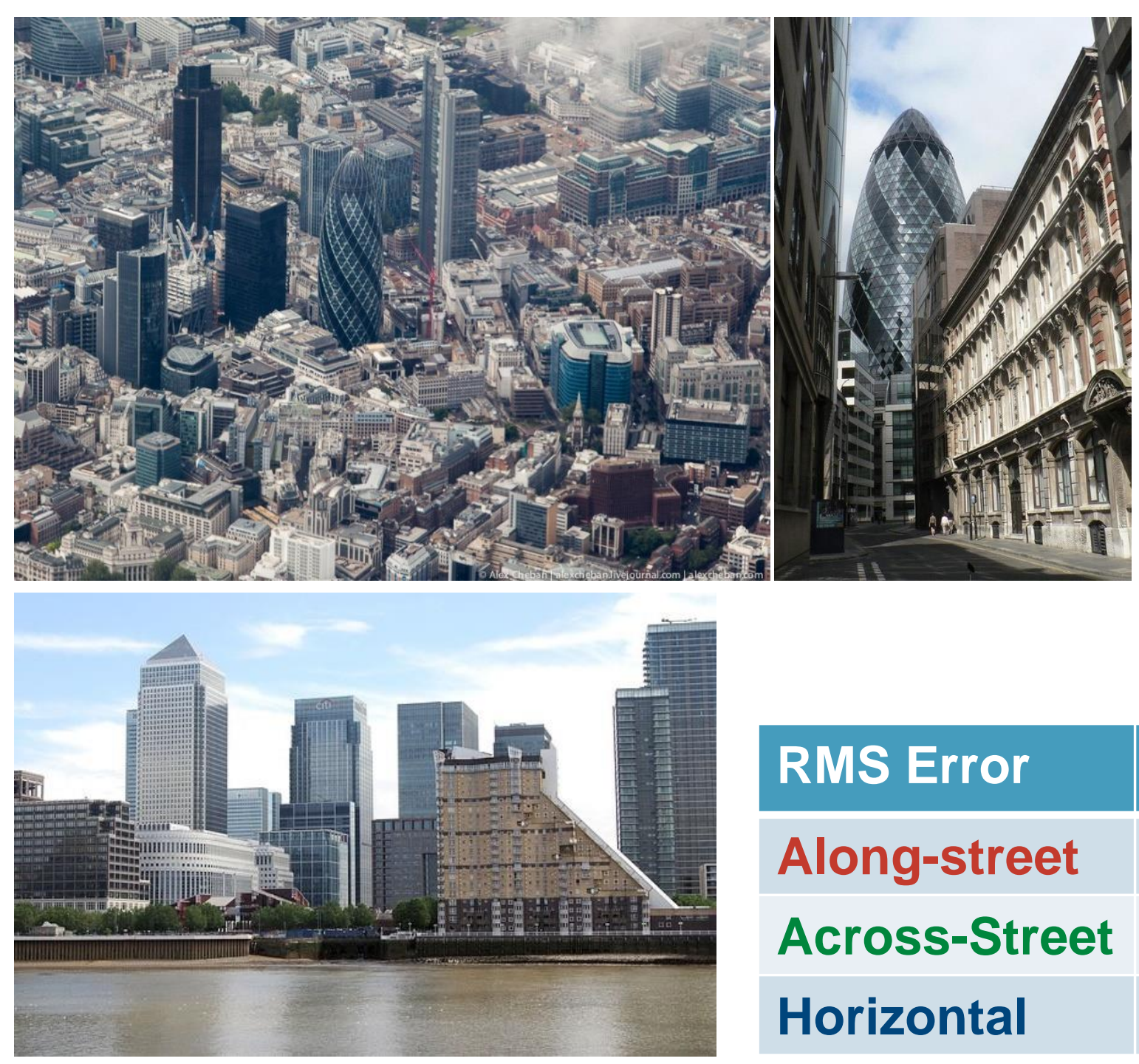

Nexus 9 Tablet Single-epoch positioning

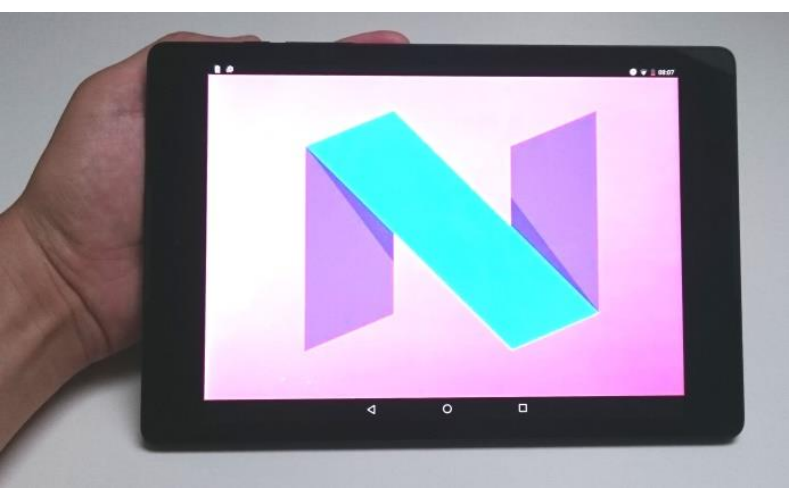

RMS Error Conventional IUP

Along-street

Across-Street

Horizontal
$17.0 \mathrm{~m}$

$28.0 \mathrm{~m}$

$32.7 \mathrm{~m}$
$4.6 \mathrm{~m}$

$5.3 \mathrm{~m}$

$7.0 \mathrm{~m}$ 


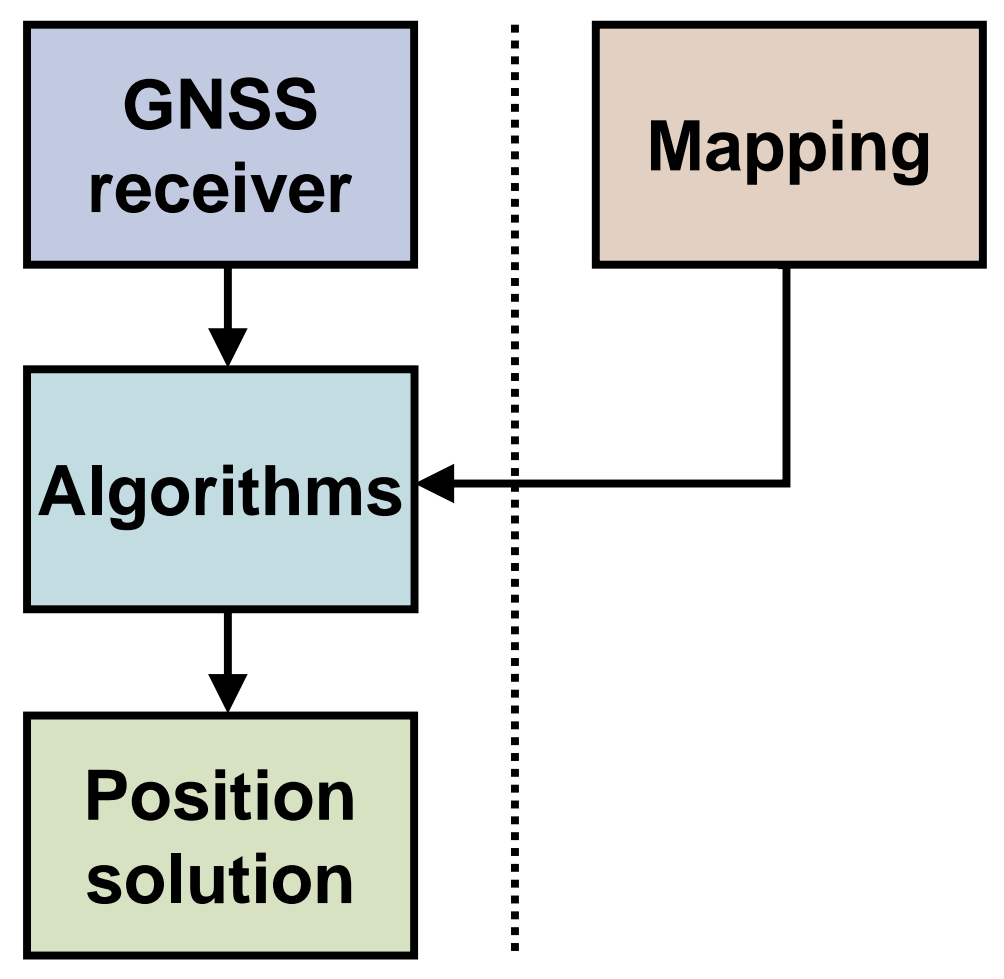

User equipment

Server
Suitable for navigation and continuous tracking applications

UCL algorithms take $233 \mathrm{~ms}$ per epoch on a laptop

Real-time implementation on a mobile device at $1-2 \mathrm{~Hz}$ is feasible

Mapping can be streamed over a $3 \mathrm{G}$ or $4 \mathrm{G}$ link

Needs new GNSS receiver interface (Android Nougat)

Would benefit from multi-epoch algorithms (planned) 


\section{Practical Implementation: Server-Based}

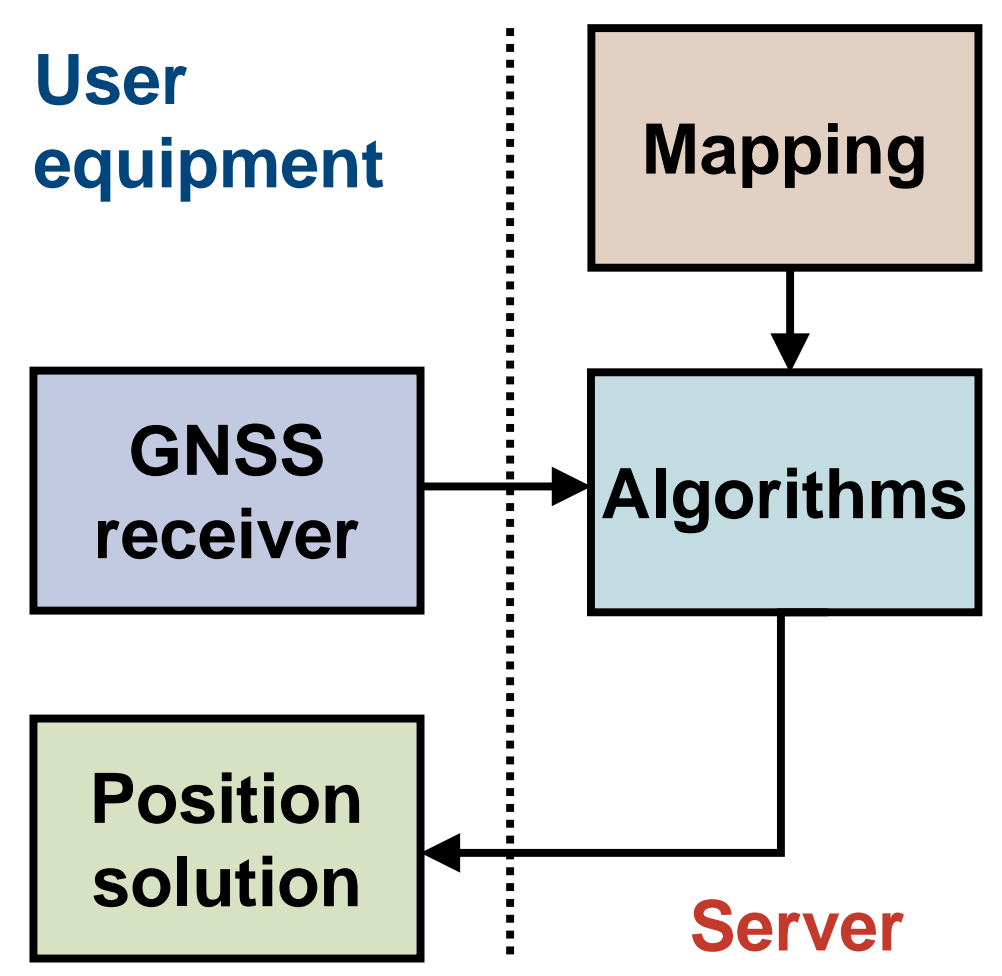

Suitable for:

- Location-based services (LBS) requiring a single fix

- Tracking applications with long update intervals

Can use existing AGNSS communications protocols, so should work with any mobile device

Suitable for current single-epoch Intelligent Urban Positioning algorithms 


\section{Summary}

\section{It's time for 3D-Mapping-Aided GNSS!}

\begin{tabular}{|l|c|c|}
\hline RMS Horizontal (2D) Position Error & Conventional & IUP \\
\hline U-blox EVK 8MT Receiver & $28.2 \mathrm{~m}$ & $4.0 \mathrm{~m}$ \\
\hline Nexus 9 Tablet (Nougat interface) & $32.7 \mathrm{~m}$ & $7.0 \mathrm{~m}$
\end{tabular}

\section{Further Information}

- Inside GNSS September/October 2016 Dr Paul Groves (UCL)

- Session A5 Presentation 7 (Friday AM) Dr Mounir Adjrad (UCL)

- Session B6 Presentation 6 (Friday PM) Dr Taro Suzuki (Waseda Uni)

Thank You to Dr Mounir Adjrad of UCL 